\title{
Effects of the sugar and fat substitution on the rheological properties of the pie dough
}

\author{
Dana Huțu, Sonia Amariei
}

Stefan cel Mare University of Suceava, Suceava, Romania

\begin{tabular}{l}
\hline Keywords: \\
Pie \\
Dough \\
Rheology \\
Sugar \\
Fat \\
Apple puree \\
\end{tabular}

Article history:

Received 30.04.2021

Received in revised

form 08.09.2021

Accepted 30.09.2021

\section{Corresponding \\ author:}

Dana Huțu

E-mail:

dana.hutu@outlook.com

DOI: $10.24263 / 2304-$

974X-2021-10-3-12

\section{Abstract}

Introduction. The aim of this research was to determine the impact on the rheological properties of the pie dough in the case of substitution of a percentage of sugar and fat with apple puree.

Materials and methods. The evaluation of the empirical rheological characteristics of the pie dough was performed using the Alveograph tool. For the evolution of the loss and storage behaviour of the dough during processing were performed two dynamic methods: frequency and creep test.

Results and discussion. The rheological properties of the doughs showed significant changes in the case of samples obtained by substituting a lower percentage of sugar and fat with apple puree. Lower values of modulus of elasticity and viscosity were obtained with the frequency of the samples obtained by substituting with puree a lower percentage of sugar and fat compared to the control sample. The sample obtained by substituting $40 \%$ of the amount of sugar and fat had the values of the viscosity module with the frequency closest to those of the control sample.

In the case of samples with a substitution of 20 and $50 \%$ of the amount of sugar and fat, the maximum gelatinization temperature had higher values than the control sample, and the samples with a substitution of 10 and $30 \%$ of the amount of sugar and fat had showed a lower maximum gelatinization temperature than the control sample. In contrast, the sample with a substitution of $30 \%$ of the amount of sugar and fat had a maximum gelatinization temperature as the control sample.

The behaviour of the dough at creep and recovery most similar to the control sample was in the case of the sample obtained by substituting $40 \%$ of the amount of sugar and fat, followed by the one with $50 \%$ substitution.

This is because the sugar and fat in apple puree have fulfilled the functions of sugar and fat added in the control sample.

Conclusions. A reduction of sugar between 10 and 50\% was achieved in 5 samples of pie dough. The use of apple puree as an ingredient in substituting a percentage of the amount of sugar and fat produced a dough with rheological properties approximately as the control sample depending on the percentage of sugar and fat substituted. 


\section{Introduction}

The pie dough displays a viscoelastic behaviour, therefore the rheological properties and its creep behaviour are followed. In addition, by substituting a quantity of sugar and fat, the dough can change its rheological properties and viscoelastic behaviour, which is the study of our research. Substituting an ingredient in the pie making process requires evaluating the behavior of the dough. The present study aimed to perform dynamic rheological measurements to evaluate the viscoelastic properties of the dough.

Dough rheology plays an essential role in baking products quality prediction and may give information about mechanical behavior (Song et al., 2007). Dynamic oscillatory measurement is a fundamental approach widely used for structural and fundamental characteristics of wheat flour dough evaluation (Sigman-Grant et al., 2005). In order to better understand the system behavior, rheological tests like creep-recovery are also required (Dobraszczyk et al, 2003) to highlight the importance of mixing energy in dough structure development. These tests can also give information about bread volume potential (Thompson et al., 2008), and dough behavior during processing (Berland et al., 1995). Dough and bread characteristics suffer modifications when dairy ingredients are added in the technological process. Therefore, it is necessary to consider certain aspects, such as the percentage added, the type of dairy ingredient and the changes that occur during the production process. The influence of dairy ingredients on dough rheology depends on the composition, and particularly on the dairy protein-gluten interaction (Mann et al., 2013; Mironeasa et al., 2018).

As regards the effect of sugar and fat on the typical rheology of dough and pie dough, sucrose is hydroscopic and therefore binds to the water found in the cake batter. This results in an increase in the viscosity of the batter which is important as this helps to retain gas bubbles, increasing the final volume of the cake. As sugar binds with water, this prevents the full hydration of the gluten proteins (found in the flour), preventing theformation of a gluten network (Perego et al., 2007). Sucrose increases the temperature of starch gelatinisation and egg protein denaturation, allowing gas bubbles to expand before the formation of the gel (Christ et al., 2005; Psimouli et al., 2012)

The objective of the present study was to investigate the effects of reducing sucrose and fat in a cake formulation and include natural sugar alternatives.

The aim of this research was to determine the impact on the rheological properties of the pie dough in the case of substitution of a percentage of sugar and fat with apple puree.

\section{Materials and methods}

\section{Sample preparation}

According to the recipe (Huțu et al., 2021), the flour: sugar ratio was 12.5:1 (g:g), and after substitution the ratio varied from 13.8:1 (in the case of a substitution of $10 \%$ of the amount of sugar with apple puree) up to 25:1 (in case of a substitution of $50 \%$ of the amount of sugar with apple puree).

In the case of fat, the ratio for the control sample was 6:1 (flour: fat, and after substitution the ratio varied from 7:1 (in the case of a substitution of $10 \%$ of the amount of fat with apple puree) to $12: 1$ (in case of a substitution of $50 \%$ of the amount of sugar with apple puree) (Huțu et al., 2021). 
Sample preparation:

$\mathrm{M}$ - sample obtained without substitution of sugar and fat,

P1 - sample obtained by substituting $10 \%$ of the amount of sugar and fat,

P2 - sample obtained by substituting $20 \%$ of the amount of sugar and fat,

P3 - sample obtained by substituting $30 \%$ of the sugar and fat content,

P4 - sample obtained by substituting $40 \%$ of the amount of sugar and fat,

P5 - sample obtained by substituting $50 \%$ of the amount of sugar and fat (Huțu et al., 2021),

\section{Rheological characteristics}

Characteristics were determined with the help of the alveographic method (Bordei et al., 2007). Each Alveograph chart was analyzed for the following parameters: $P$, the maximum pressure needed to blow the dough bubble, expresses dough resistance; $L$, length of the curve, expresses dough extensibility; $P / L$, configuration ratio of the Alveograph curve; $G$, index of swelling; $W$, baking strength (surface area of the curve).

The alveographic curve allows to determine a series of rheological characteristics of the dough:

- The maximum pressure, $\mathrm{P}(\mathrm{mm})$, expresses the resistance to deformation of the dough;

- Length, L (mm), expresses the extensibility of the dough. It shows the ability of the dough to form bubbles, until it breaks;

- The swelling index, $G$, is the average of the swelling indices of the five samples. Similar to $\mathrm{L}, \mathrm{G}$ is the expression of the extensibility of the dough;

- Conformation ratio (shape) of the curve, P / L, expresses the ratio between the elastic properties and the viscous properties of the dough;

- The deformation energy, W, expresses the energy required to inflate the dough bubble before it breaks (Bordei et al., 2007).

\section{Dough Rheological Properties}

The fundamental rheological characteristics of dough were assessed by the dynamic oscillatory method in the linear viscoelastic region. For this purpose, a HAAKE MARS 40 stress controlled rheometer (Thermo-HAAKE, Karlsruhe, Germany) with smooth parallel plates measuring system and a gap width of $2 \mathrm{~mm}$ were used. The dough samples were formulated without yeast, at the optimum water absorption capacity previously determined. Dough formulations rested for 5 min to allow relaxation and temperature stabilization, then were placed between the plates and kept for $120 \mathrm{~s}$ prior testing. The measuring temperatures were controlled by using an external thermostatic bath during the tests. The excess of dough was removed, and a Vaseline layer was applied to the exposed edge of dough, to prevent the loss of moisture during testing. Before analysis, dough samples were tested for the limits of the linear viscoelastic region (LVR) based on the strain sweep determination, in which the strain was increased from 0.01 to $1 \%$, at a constant oscillation frequency of $1 \mathrm{~Hz}$ (Iuga et al., 2020). The oscillatory measurements were performedat a maximum strain of $0.15 \%$, which was found to be in the LVR where dough samples have a linear relationship between stress and strain. 


\section{- Food Technology}

\section{Frequency Sweep Test}

The frequency sweep test was performed at a frequency variation from 1 to $20 \mathrm{~Hz}$, with a constant stress previously established, in the LVR, at a temperature of $20{ }^{\circ} \mathrm{C}$. The changes of the storage modulus $\left(\mathrm{G}^{\prime}\right)$, loss modulus $\left(\mathrm{G}^{\prime \prime}\right)$ and complex modulus $\left(\mathrm{G}^{*}\right)$ were registered. The experimental data were fitted to the Power law model using the Equations (1)-(3) (Mironeasa et al., 2019). The loss tangent $\left(\tan \delta\right.$ ) was calculated as the ratio between $G^{\prime \prime}$ and and $\mathrm{G}^{\prime}$.

$$
\begin{aligned}
& G^{\prime}(\omega)=K^{\prime} \cdot \omega^{n^{\prime}} \\
& G^{\prime \prime}(\omega)=K^{\prime \prime} \cdot \omega^{n^{\prime \prime}} \\
& G^{*}(\omega)=K^{*} \cdot \omega^{n^{*}}
\end{aligned}
$$

where $\mathrm{G}^{\prime}$ is the storage modulus $(\mathrm{Pa}), \mathrm{G}^{\prime \prime}$ loss modulus $(\mathrm{Pa}), \mathrm{G}^{*}$ complex modulus $(\mathrm{Pa}), \omega$ angular frequency $(\mathrm{rad} / \mathrm{s}), \mathrm{K}^{\prime}, \mathrm{K}^{\prime \prime}, \mathrm{K}^{*}\left(\mathrm{~Pa} \cdot \mathrm{s}^{\mathrm{n}}{ }^{\prime}\right)$ are consistency indices, $\mathrm{n}^{\prime}, \mathrm{n}^{\prime \prime}$ and $\mathrm{n}^{*}$ are flow behaviour indices (Bordei et al., 2007).

\section{Creep and Recovery Test}

Creep-recovery tests were performed by applying a constant shear stress of $50 \mathrm{~Pa}$ over a creep time of $60 \mathrm{~s}$ and allowing strain recovery during $180 \mathrm{~s}$ after stress removal, at $20{ }^{\circ} \mathrm{C}$ temperature. The compliancerheological parameter (Steffe, 1996; Lupi et al., 2020) was recorded (Equation (4)):

$$
J(t)=\frac{\gamma(t)}{\sigma}
$$

where $J\left(\mathrm{~Pa}^{-1}\right)$ is the compliance, $\gamma$ - the strain and $\sigma$ - the constant stress applied $\left(\mathrm{Pa}^{-1}\right)$ (Iuga et al., 2020).

The experimental data of creep-recovery test were fitted to the Burgers model, made up of four components, which comprises the association in series of the Maxwell model and the Kelvin-Voigt model [23,24].

The percentage recovery, which highlighted the relative elastic part of the maximum creep compliance, was also determined as the ratio between $\mathrm{J}_{\max }$ and $\mathrm{J}_{\mathrm{r}}$ (Barnes, 2000).

\section{Temperature Sweep Test}

The temperature sweep test was performed at a constant strain of $0.15 \%$ and a frequency of $1 \mathrm{~Hz}$, the dough samples being heated from 20 to $100{ }^{\circ} \mathrm{C}$ at a rate of $4.0 \pm 0.1{ }^{\circ} \mathrm{C}$ per min. The storage $\left(G^{\prime}\right)$ and loss modulus $\left(G^{\prime}\right.$ ') were recorded as a function of temperature, allowing the determination of maximumgelatinization temperatures $\left(T_{\max }\right)$ (Iuga et al., 2020). 


\section{Results and discussion}

\section{Rheological characteristics}

The table includes the numerical values of the rheological characteristics of the pie dough obtained by substituting in different percentages the sugar and fat with apple puree.

Rheological characteristics of pie dough

Table 1

\begin{tabular}{|l|l|l|l|l|l|l|}
\hline Characteristics & M & P1 & P2 & P3 & P4 & P5 \\
\hline P $(\mathrm{mm})$ & 80 & 58 & 62 & 73 & 106 & 76 \\
\hline L $(\mathrm{mm})$ & 110 & 112 & 125 & 78 & 74 & 91 \\
\hline G & 23,3 & 23,6 & 24,9 & 19,7 & 19,1 & 21,2 \\
\hline W $\left(10^{-4} \mathrm{j}\right)$ & 234 & 155 & 187 & 168 & 253 & 190 \\
\hline P/L & 0,73 & 0,52 & 0,50 & 0,94 & 1,43 & 0,84 \\
\hline
\end{tabular}

Where:

M-sample obtained according to the manufacturing recipe without substitution of sugar and fat, P1 - sample obtained by substituting $10 \%$ of the amount of sugar and fat;

P2 - sample obtained by substituting $20 \%$ of the amount of sugar and fat;

P3 - sample obtained by substituting $30 \%$ of the amount of sugar and fat;

P4 - sample obtained by substituting $40 \%$ of the amount of sugar and fat;

P5 - sample obtained by substituting 50\% of the amount of sugar and fat;

$\mathrm{P}$ - maximum pressure;

L - dough extensibility;

W - baking strength;

$\mathrm{G}$-index of swelling;

$\mathrm{P} / \mathrm{L}$ - configuration ratio of the Alveograph curve.

This is because sucrose is hydroscopic and therefore binds to the water found in the cake batter. This results in an increase in the viscosity of the batter which is important as this helps to retain gas bubbles, increasing the final volume of the cake. As sugar binds with water, this prevents the full hydration of the gluten proteins (found in the flour), preventing theformation of a gluten network (Perego et al., 2007). Sucrose increases the temperature of starch gelatinisation and egg protein denaturation, allowing gas bubbles to expand before the formation of the gel (Christ et al., 2005; Psimouli et al., 2012).

Regarding the maximum pressure (Figure 2c) exerted on the pie dough, which expresses its deformation resistance, it was found, following the determinations, that they will be obtained by substituting 10 and $20 \%$ of the amount of sugar and fat have a lower deformation resistance. compared to the control sample, the samples obtained by substituting $30 \%$ and $50 \%$ of the amount of sugar and fat have a deformation resistance similar to the control sample, whereas the sample obtained by substituting $40 \%$ of the amount of sugar and fat has a significantly higher deformation resistance than the control sample.

The capacity of the dough to form bubbles until breaking is expressed by its extensibility (Figure 2a), which in the case of the sample in which the amount of sugar was replaced by $10 \%$ is closest to the value of the control. In the case of the sample in which the replacement was $20 \%$, the extensibility showed higher values, and in the case of samples with a replacement of 30, 40 and $50 \%$ the extensibility is significantly lower. 


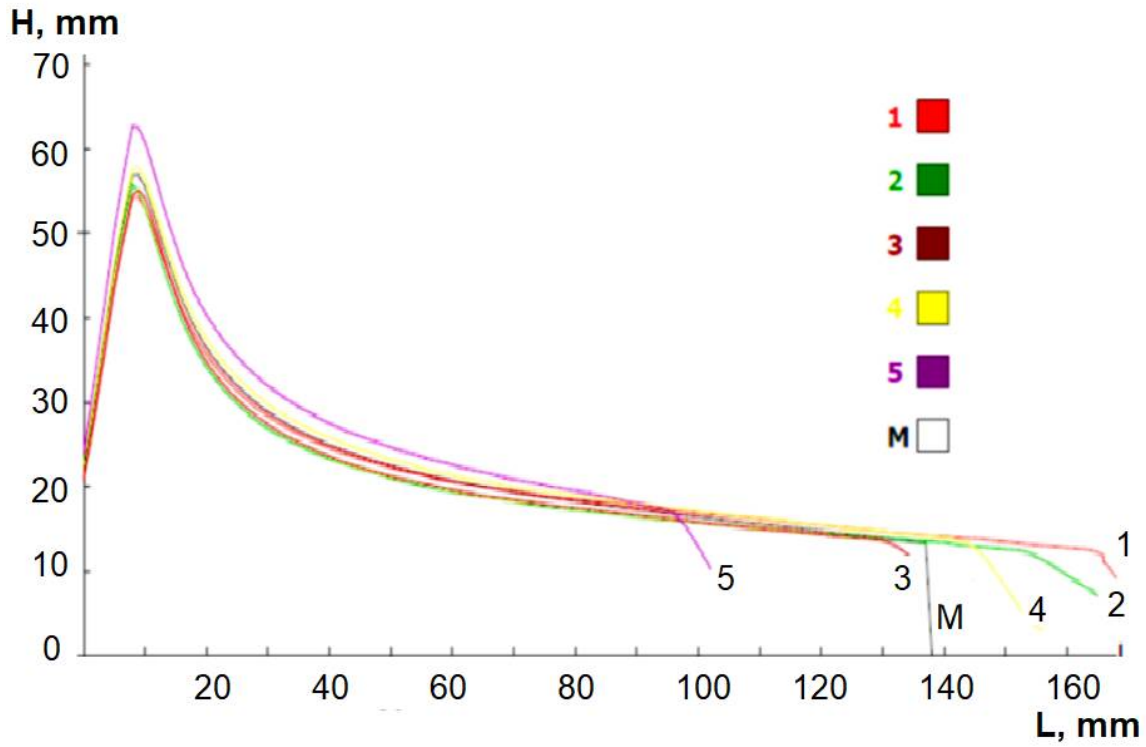

Figure 1. Alveographic curve for pie dough:

$\mathrm{M}$ - sample obtained according to the manufacturing recipe without substitution of sugar and fat;

P1 - sample obtained by substituting $10 \%$ of the amount of sugar and fat; P2 - sample obtained by substituting $20 \%$ of the amount of sugar and fat; P3 - sample obtained by substituting $30 \%$ of the amount of sugar and fat; P4 - sample obtained by substituting $40 \%$ of the amount of sugar and fat, P5 - sample obtained by substituting $50 \%$ of the amount of sugar and fat.

As in the case of extensibility, the swelling index, as an expression of extensibility, showed a similar rheological behavir as in the case of extensibility. In this case, the values of the samples obtained by substituting in different proportions the sugar with apple puree, respectively 10, 20,30, 40 and 50\%, the swelling index (Figure 2b) does not show significant variations compared to the control sample obtained according to the manufacturing recipe of pie dough. 


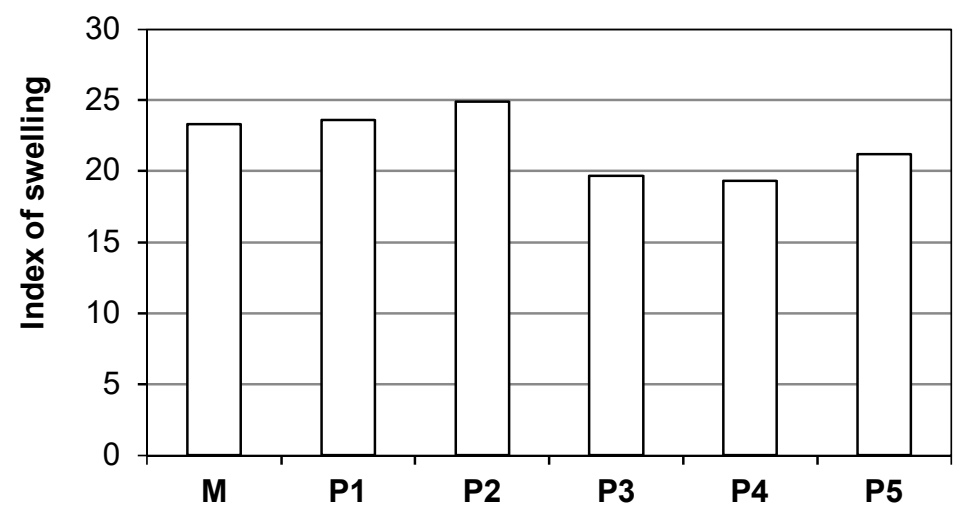

a

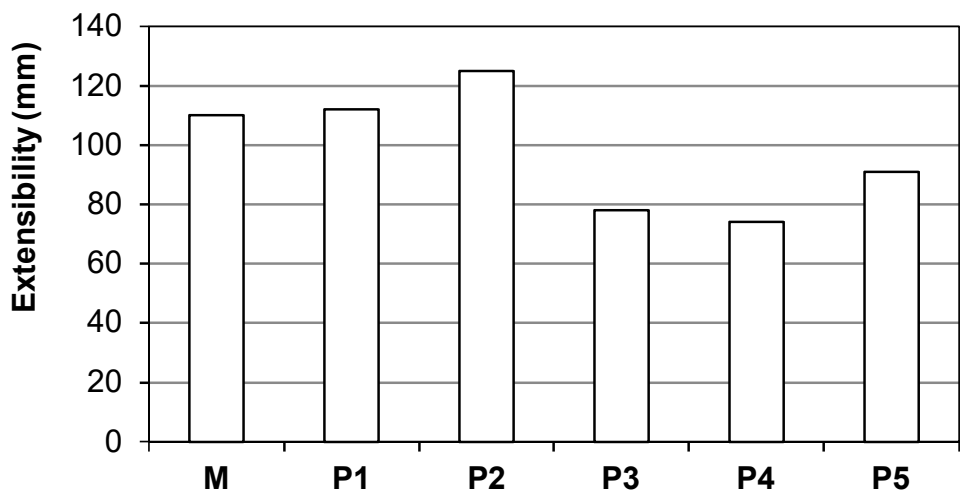

b

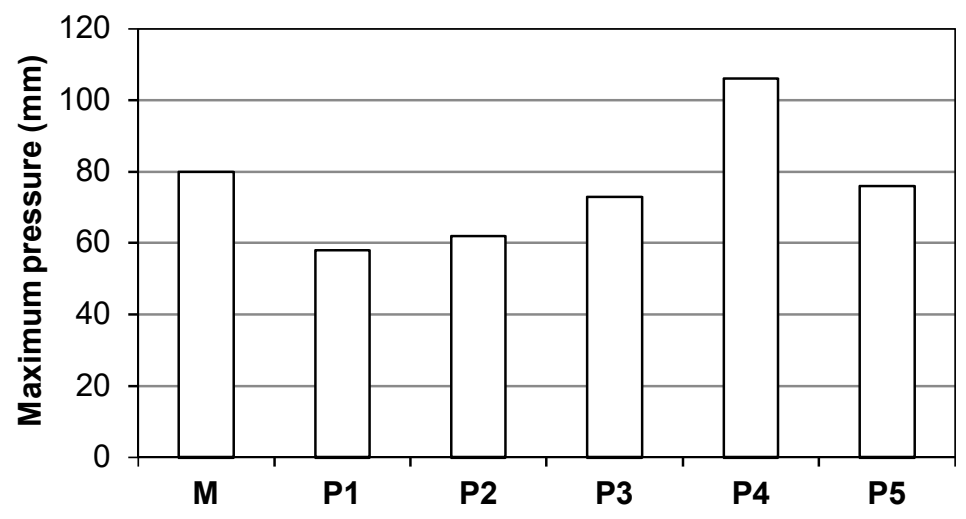

Figure 2. Graphical representation of the swelling index (a), extensibility (b) and maximum pressure (c).

Alveographic curve for pie dough:

$\mathrm{M}$ - sample obtained according to the manufacturing recipe without substitution of sugar and fat;

P1 - sample obtained by substituting $10 \%$ of the amount of sugar and fat;

P2 - sample obtained by substituting $20 \%$ of the amount of sugar and fat;

P3 - sample obtained by substituting $30 \%$ of the amount of sugar and fat;

P4 - sample obtained by substituting $40 \%$ of the amount of sugar and fat,

P5 - sample obtained by substituting $50 \%$ of the amount of sugar and fat. 


\section{Frequency Sweep Test}

The frequency sweep tests data obtained showed that, in the range of the considered frequencies, the mechanical spectra of the storage modulus $\left(G^{\prime}\right)$ (Figure 3) was greater than of the loss modulus (G") for the control, the samples samples obtained by substituting sugar and fat with apple puree, indicating that the dough have a viscoelastic behavior, as was expected (Figure 4).

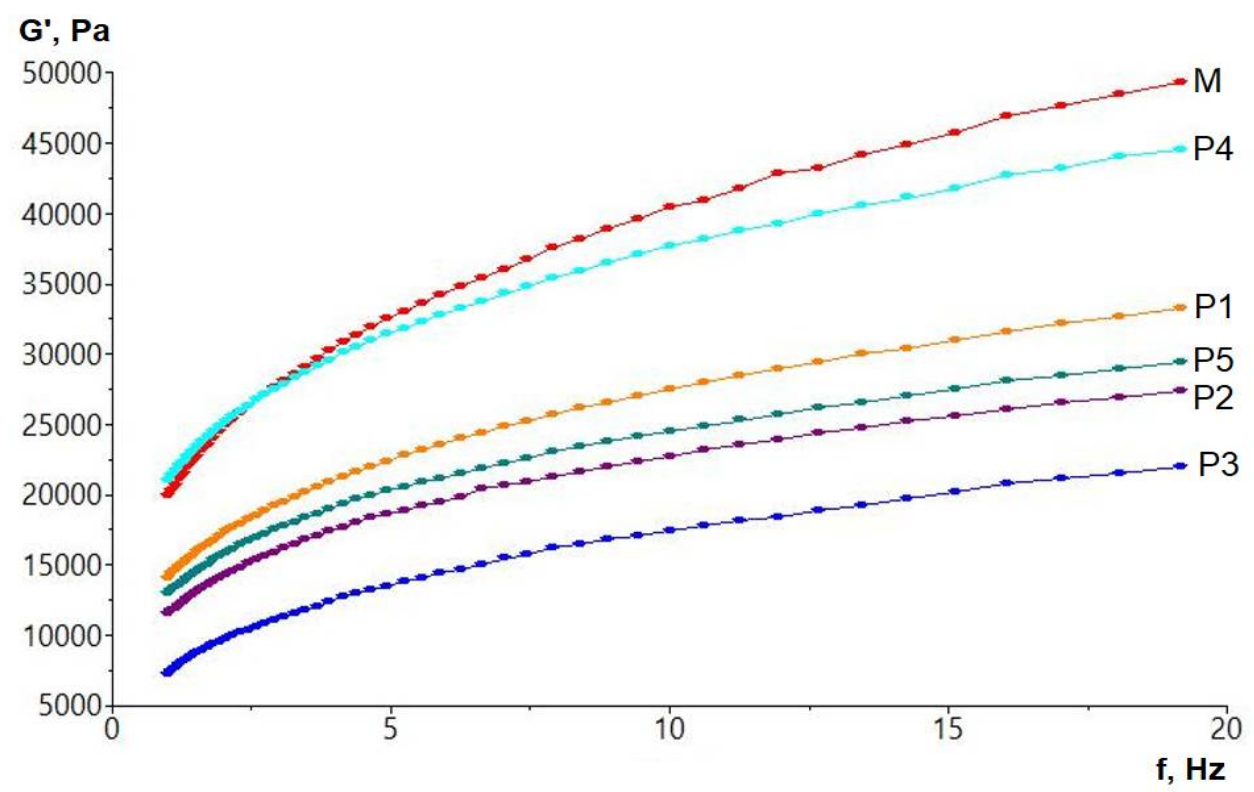

Figure 3. Storage modulus (G') variations with frequency:

$\mathrm{M}$ - sample obtained according to the manufacturing recipe without substitution of sugar and fat;

P1 - sample obtained by substituting $10 \%$ of the amount of sugar and fat; P2 - sample obtained by substituting $20 \%$ of the amount of sugar and fat; P3 - sample obtained by substituting $30 \%$ of the amount of sugar and fat; P4 - sample obtained by substituting $40 \%$ of the amount of sugar and fat, P5 - sample obtained by substituting 50\% of the amount of sugar and fat.

The tested samples exhibited a predominant elastic solid-like behavior $\left(G^{\prime}>G^{\prime \prime}\right)$ over the entire experimental frequency range. The $\mathrm{G}^{\prime}$ and $\mathrm{G}^{\prime \prime}$ moduli increased with the frequency increase, which means that dough recovery after a stress application was slow, due to the fact that the network is not completely elastic.

The replacement of sugar and fat with apple puree to a significant $(p<0.001)$ increase of storage and loss moduli compared to the control, except for sample obtained by replacing $40 \%$ of the amount of sugar and fat with apple puree. 


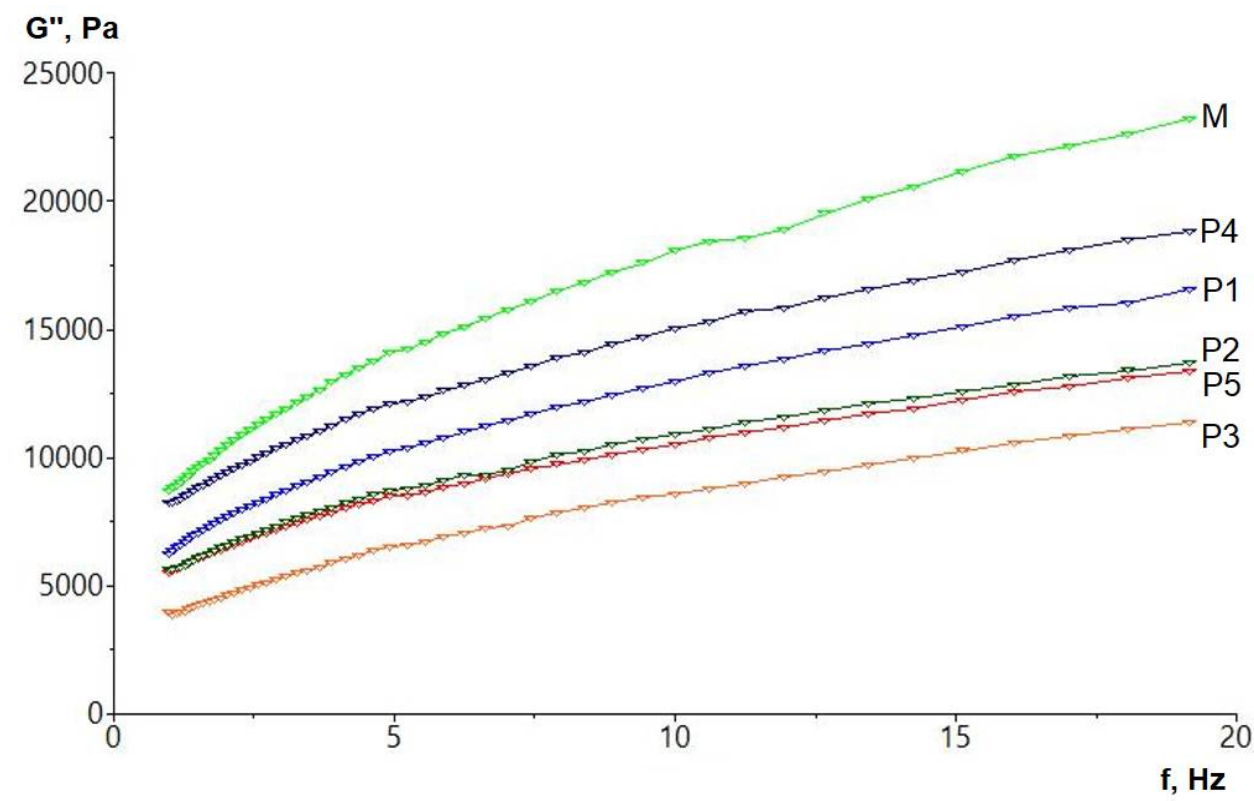

Figure 4. Loss modulus (G”) variations with frequency:

$\mathrm{M}$ - sample obtained according to the manufacturing recipe without substitution of sugar and fat;

P1 - sample obtained by substituting $10 \%$ of the amount of sugar and fat;

P2 - sample obtained by substituting $20 \%$ of the amount of sugar and fat;

P3 - sample obtained by substituting $30 \%$ of the amount of sugar and fat;

P4 - sample obtained by substituting $40 \%$ of the amount of sugar and fat,

P5 - sample obtained by substituting 50\% of the amount of sugar and fat.

\section{Temperature Sweep Test}

The storage modulus $\left(G^{\prime}\right)$ variation with temperature are shown in Figure 5. At first, the storage modulus $\left(\mathrm{G}^{\prime}\right)$ appears at a minimum level up to a certain temperature, then it suddenly increases until the maximum gelatinization temperature is reached, then decreases again. Samples obtained by substituting 20 and $50 \%$ of the amount of sugar and fat showed higher gelatinization temperatures compared to the control, samples obtained by substituting $10 \%$, $30 \%$ of the amount of sugar and fat showed higher gelatinization temperatures low compared to the control, and the sample obtained by substituting $40 \%$ of the amount of sugar and fat showed the same value of the gelatinization temperature as the control (Figure 4). The peak values of the storage modulus $\mathrm{G}^{\prime}$ were significantly higher for the samples obtained by substituting 20 and $50 \%$ of the amount of sugar and fat. 


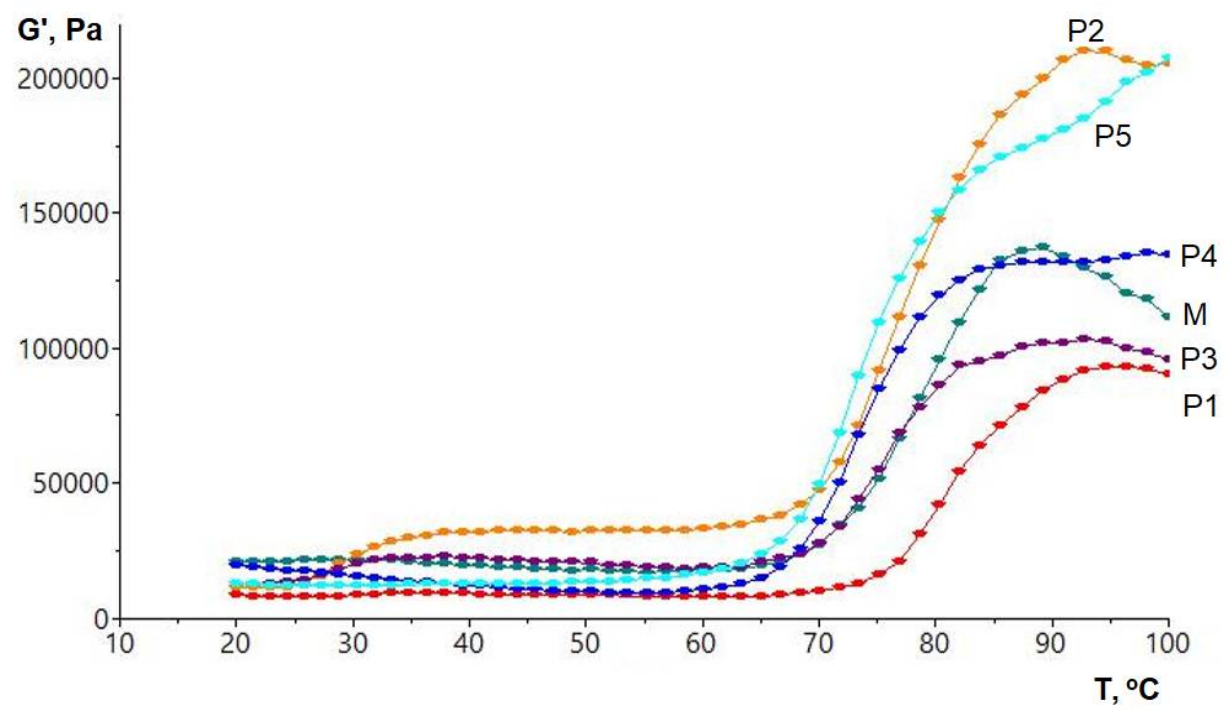

Figure 5. Storage modulus (G' ) variations with temperature

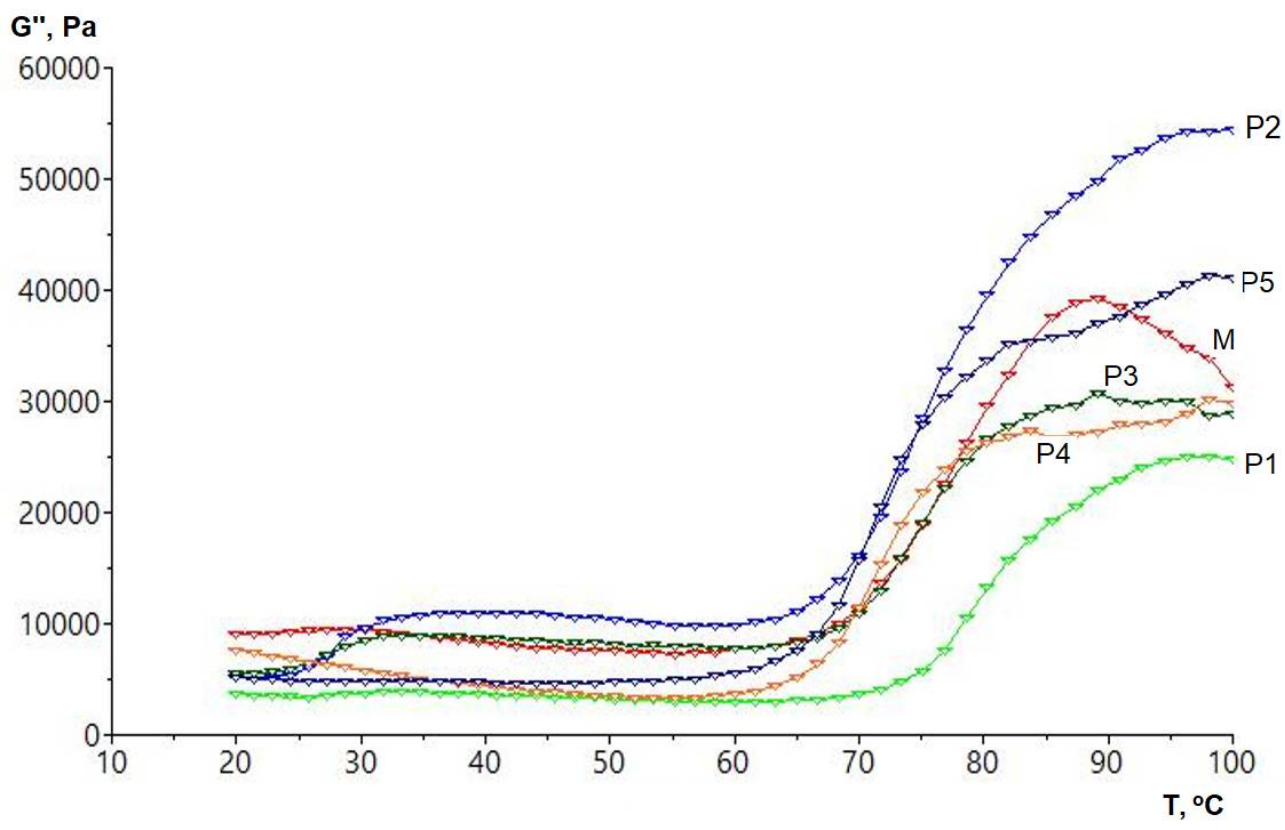

Figure 6. Loss modulus (G', ) variations with temperature

For figures 5 and 6:

$\mathrm{M}$ - sample obtained according to the manufacturing recipe without substitution of sugar and fat;

P1 - sample obtained by substituting $10 \%$ of the amount of sugar and fat;

P2 - sample obtained by substituting $20 \%$ of the amount of sugar and fat;

P3 - sample obtained by substituting $30 \%$ of the amount of sugar and fat;

P4 - sample obtained by substituting $40 \%$ of the amount of sugar and fat,

P5 - sample obtained by substituting 50\% of the amount of sugar and fat. 
The loss modulus $\left(\mathrm{G}^{\prime \prime}\right)$ variation with temperature are shown in Figure 6.

At first, the loss modulus (G") appears at a minimum level up to a certain temperature, then it increases sharply until the maximum gelatinization temperature is reached, then decreases again. Samples obtained by substituting 20 and $50 \%$ of the amount of sugar and fat showed higher gelatinization temperatures compared to the control, samples obtained by substituting 10,30 and $40 \%$ of the amount of sugar and fat showed temperatures lower gelatinization compared to the control (Figure 4). The peak values of the loss modulus $\mathrm{G}^{\prime}$ were significantly lower for the samples obtained by substituting 20,30 and $40 \%$ of the amount of sugar and fat

\section{Creep and Recovery Test}

According to the results shown in Figure 7, the replacement of sugar and fat with apple puree clearly influenced the storage and loss properties of the doughs.

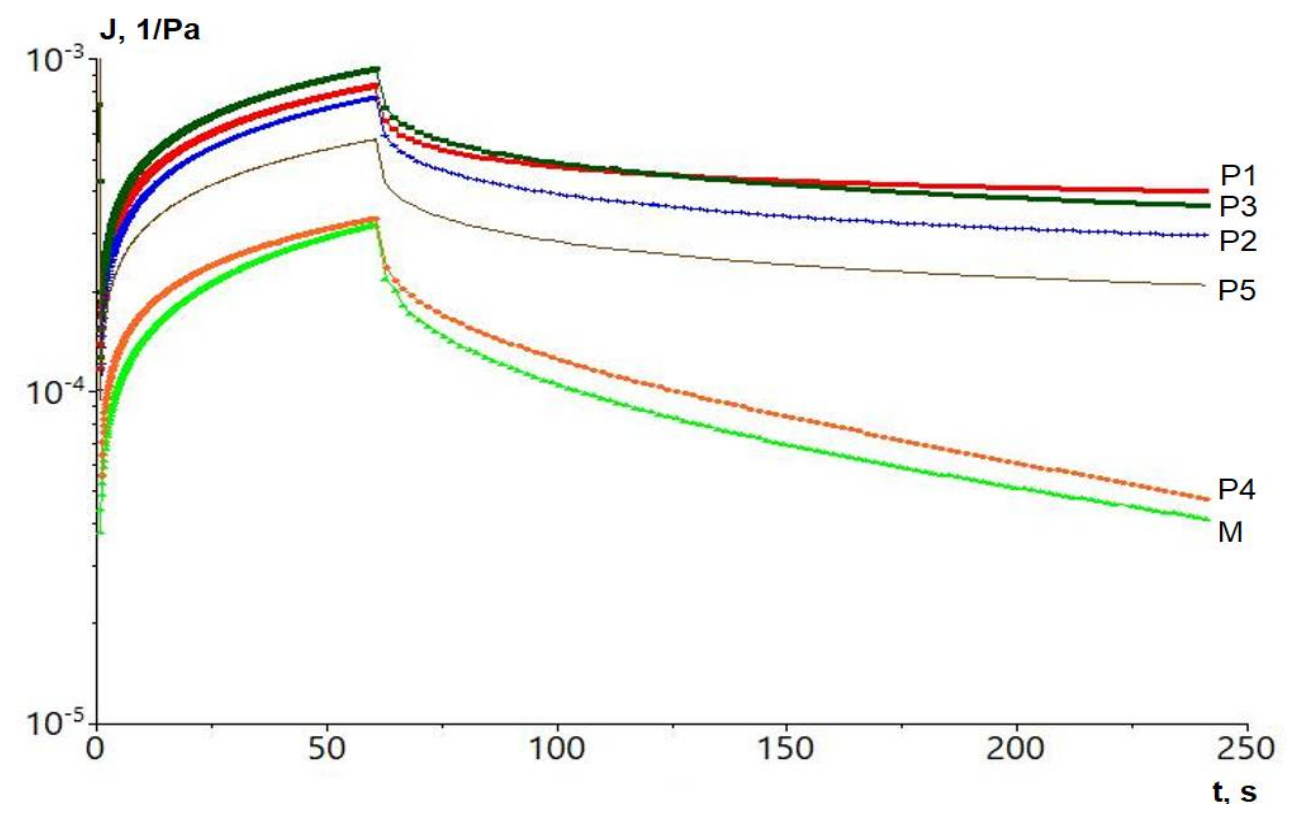

Figure 7. Creep and recovery curves of pie dough:

$\mathrm{M}$ - sample obtained according to the manufacturing recipe without substitution of sugar and fat; P1 - sample obtained by substituting $10 \%$ of the amount of sugar and fat; P2 - sample obtained by substituting $20 \%$ of the amount of sugar and fat; P3 - sample obtained by substituting $30 \%$ of the amount of sugar and fat; P4 - sample obtained by substituting $40 \%$ of the amount of sugar and fat, P5 - sample obtained by substituting 50\% of the amount of sugar and fat.

The doughs obtained by replacing $10,20,30$ and $50 \%$ of the amount of sugar and fat (P1, P2, P3) showed significantly higher conformity values during the creep test (Figure 7). The sample obtained by replacing sugar and fat in a proportion of $40 \%$ showed a creep and return behavior almost identical to that of the control sample. Compared to the control 
sample, the instantaneous $\left(\mathrm{J}_{\mathrm{Co}}, \mathrm{J}_{\mathrm{Ro}}\right)$ and retarded $\left(\mathrm{J}_{\mathrm{Co}}, \mathrm{J}_{\mathrm{Ro}}\right)$ compliances for both the creep and the recovery phase were similar to the control are those of the sample obtained by substituting $40 \%$ of the the amount of sugar and fat, higher for the sample obtained by substituting $50 \%$ of the amount of sugar and fat and significantly higher than the samples obtained by substituting $10 \%, 20 \%, 30 \%$ of the amount of sugar and fat, which indicated a moreinstantaneous and retarded deformation increased.

\section{Conclusion}

Replacing some quantity of sugar and fat with apple puree has led to an increase in the nutritional, functional and technological value of pastries. The nutritional value has increased due to the content of nutrients, especially fiber. A reduction of sugar and fat between 10 and $50 \%$ was achieved in 5 samples of pie dough. The use of apple puree as an ingredient in substituting a percentage of the amount of sugar and fat produced a dough rheological properties approximately as the control sample depending on the percentage of sugar and fat substituted.

The rheological properties of the dough showed significant changes even in the case of a lower puree substitution percentage of sugar and fat. Lower values of modulus of elasticity and viscosity were obtained with the frequency of the samples obtained by substituting with puree a lower percentage of sugar and fat. Compared to the control sample, the sample obtained by substituting $40 \%$ of the amount of sugar and fat had the values of the modulus of viscosity and elasticity with frequency, respectively the values of the modulus of viscosity and elasticity with the temperature closest to those of the control sample.

In the case of samples with a substitution of 20 and $50 \%$ of the amount of sugar and fat, the maximum gelatinization temperature had higher values than the control sample, and the samples with a substitution of 10 and $30 \%$ of the amount of sugar and fat had showed a lower maximum gelatinization temperature than the control sample. In contrast, the sample with a substitution of $30 \%$ of the amount of sugar and fat had a maximum gelatinization temperature as the control sample.

The behaviour of the dough at creep and recovery most similar to the control sample was in the case of the sample obtained by substituting $40 \%$ of the amount of sugar and fat, followed by the one with $50 \%$ substitution.

\section{References}

Barnes H.A. (2000), Handbook of Elementary Rheology, Institute of Non-Newtonian Fluid Mechanics, Aberystwyth, Wales.

Berland S., Launay B. (1995), Shear softening and thixotropic properties of wheat flour doughs in dynamic testing at high shear strain, Rheola Acta, 34, pp. 622-625.

Bordei D., Bahrim G., Pâslaru V., Gasparotti C., Elisei A., Banu I., Ionescu L., Codină G. (2007), Controlul calităţii în industria Panificației - Metode de analiză, Editura Academica, Galați, pp. 378-387.

Christ D., Takeuchi K. P., Cunha R. L. (2005), Effect of sucrose addition and heat treatment on egg albumen protein gelation, Journal of Food Science, 70(3), E230-E238.

Dobraszczyk B.J., Morgenstern M.P. (2003), Rheology and the breadmaking process, $J$. Cereal Sci., 38, pp. 229-245. 
Huțu D.. Amariei S. (2021), The effects of sugar and fat substitution on the textural properties of the pie dough, Food and Environment Safety - Journal, XX(2), pp. 149-159.

Iuga M., Boestean O., Ghendov-Mosanu A., Mironeasa S. (2020), Impact of dairy ingredients on wheat flour dough rheology and bread properties, Foods, 9(6), p. 828.

Lupi F.R., Puoci F., Bruno E., Baldino N., MarinoR., Gabriele, D. (2020), The effects of process conditions on rheological properties of functional citrus fibre suspensions. Food and Bioproducts Processing, 121, pp. 54-64.

Mann J., S. Mallard, L. Te Morenga (2013), Dietary sugars and body weight: Systematic review and meta-analyses of randomised controlled trials and cohort studies, $B M J, 345$, e7492.

Mironeasa S., Iuga M., Zaharia D., Mironeasa C. (2019), rheological analysis of wheat flour dough as influenced by grape peels of di_erent particle sizes and addition levels, Food and Bioprocess Technology, 12, pp. 228-245.

Mironeasa S., Zaharia D., Codină G. G., Ropciuc S., \& Iuga M. (2018), Effects of grape peels addition on mixing, pasting and fermentation characteristics of dough from 480wheat flour type, Bulletin of University of Agricultural Sciences and Veterinary Medicine Cluj-Napoca Food Science and Technology, 75, 27.

Perego P., Sordi A., Guastalli R., \& Converti A. (2007), Effects of changes in ingredient composition on the rheological properties of a biscuit industry dough, International Journal of Food Science and Technology, 42(6), pp. 649-657.

Psimouli V., \& Oreopoulou V. (2012), The effect of alternative sweeteners on batter rheology and cake properties, Journal of the Science of Food and Agriculture, 92(1), pp. 99-105.

Sigman-Grant, M. J., G. Hsieh (2005), Reported use of reducedsugar foods and beverages reflect high-quality diets, Journal of Food Science, 70, S42-S46.

Song Y., Zheng Q. (2007), Dynamic rheological properties of wheat flour dough and proteins, Trends in Food Science and Technology, 18, pp. 132-138.

Steffe J.F. (1996), Rheological Methods, Food Process Engineering, Freeman Press, East Lansing, Michigan.

Thompson A. K., Moughan P. J. (2008), Innovation in the foods industry: Functional foods. Innovation, 10(1), pp. 61-73. 